\title{
Trastorno de estrés postraumático y dolor crónico: nexos entre psico y pato(logía)
}

\author{
Alicia E. López Martínez, Lydia Gómez Pérez, Gema T. Ruiz Párraga \\ Dpto. Personalidad, Evaluación y Tratamiento Psicológico. Universidad de Málaga
}

Disponible online 22 diciembre de 2009

\begin{abstract}
El objetivo primordial de este artículo es efectuar una breve revisión acerca de la literatura existente en torno a la investigación sobre la relación entre dolor crónico y el trastorno por estrés postraumático (TEPT). Existe un creciente cuerpo de evidencia empírica indicativo de que el TEPT y el dolor crónico co-ocurren frecuentemente, y de que ambos problemas interaccionan, de modo tal que pueden afectarse mutuamente e incidir negativamente en el curso y pronóstico del tratamiento aplicado a uno y otro. De hecho, las personas que sufren ambos problemas de salud manifiestan mayor malestar y discapacidad comparados con quienes tan sólo padecen uno de los dos trastornos. De ahí que en los últimos años se han venido proponiendo modelos conceptuales y mecanismos potencialmente explicativos sobre cómo y por qué ambos trastornos interaccionan. La investigación al respecto sugiere que variables como el miedo y la evitación, la sensibilidad a la ansiedad y el castastrofismo, así como ciertas características psicológicas y biológicas, específicas y generales, pueden contribuir a explicar el desarrollo y mantenimiento de estos problemas.
\end{abstract}

Palabras clave: trastorno por estrés postraumático, dolor crónico, comorbilidad, mecanismos explicativos

The primary aim of this paper is to provide a brief review of the existing literature investigating the relationship between chronic pain and the Posttraumatic Stress Disorder (PSTD). There is a growing body of research that indicates that PSTD and chronic pain frequently co-occur, and that both conditions may interact in such a way as to negatively impact the course and outcome of the treatment of either disorder. People with both health problems tend to have greater distress and impairment compared to those with only one of these conditions. In recent years, several important conceptual developments and potential mechanisms have been proposed on how and why these disorders often co-occur and how they can interact with one another. The research suggests that variables such as fear and avoidance, anxiety sensitivity, and catastrophizing, as well as general and specific psychological and biological features, may help account for the development and maintenance of both conditions.

Keywords: posttraumatic stress disorder, chronic pain, comorbidity, explaining mechanisms.

Correspondencia: Alicia E. López Martínez. Dpto. Personalidad, Evaluación y Tratamiento Psicológico. Facultad de Psicología. Universidad de Málaga. Campus de Teatinos, s/n. 29071-Málaga. Fax: 9521311 01. E-mail: aelm@uma.es 
Es cada vez mayor el interés por analizar los mecanismos que explican la relación entre el trastorno por estrés postraumático y el dolor crónico. La evidencia tanto empírica como clínica pone de manifiesto la existencia de una clara comorbilidad entre ambos mostrando, incluso, que pueden interactuar hasta el punto de afectar el curso y resultado del tratamiento aplicado a uno y otro caso. Lo que subyace como explicación a esta interacción es la hipótesis de que ambos problemas comparten mecanismos que explican su mantenimiento. Las páginas que siguen tienen como propósito exponer brevemente el estado actual de la investigación al respecto.

El padecimiento de trastorno de estrés postraumático como precursor del deterioro de la salud física

Es bien sabido que la vivencia de experiencias traumáticas tiene numerosas consecuencias para el organismo, no sólo psicológicas sino también físicas. Diversos estudios han venido mostrando que el trastorno de estrés postraumático, causado por la exposición a situaciones traumáticas de diversa índole (guerras, catástrofes naturales, abuso físico y sexual, o violencia de género), se asocia a la presencia de síntomas físicos variados así como a una mayor morbilidad, destacando entre ellas la patología cardiovascular (Boscarino, 1997; Boscarino y Chang, 1999; Falger et al., 1992; Shemesh et al., 2004; Sawchuk et al., 2005) y las enfermedades musculoesqueléticas que cursan con dolor (Cwikerl, Abdelgani, Goldsmiths, Quastel y Yevelson, 1997; Dobie et al., 2004; McFarlane, Atchison, Rafalowicz y Papay, 1994).

El reconocimiento del trastorno por estrés postraumático (TEPT, en adelante) como entidad nosológica es relativamente reciente, si se tiene en consideración que es en 1980 cuando se recoge como tal en el Manual Diagnóstico y Estadístico de Trastornos Mentales -habitualmente denominado DSM- editado por la Asociación de Psiquiatría Americana (APA, 1980). Probablemente, aunque este trastorno ya se comenzó a estudiar en el siglo XIX, la mayor presencia de conflictos bélicos en el siglo XX y, particularmente, la Guerra de Vietnam de mediados de 1960 son los responsables del interés por el TEPT y de su consideración como una categoría diagnóstica oficial (Commitee on Treatment of Post-Traumatic Stress Disorder, 2008).

Actualmente, el DSM-IV define el TEPT a partir de los siguientes criterios (APA, 2000): a) haber sufrido (o haber sido testigo de) una experiencia traumática que implique una amenaza para la integridad física propia o de los demás; b) reaccionar ante dicha experiencia con un miedo intenso, con indefensión o con horror; c) experimentar recuerdos o imágenes intrusivos del acontecimiento traumático; d) evitar situaciones asociadas al acontecimiento traumático y paralización ante las mismas; e) responder con una marcada hiperactivación (arousal elevado), lo que se ve reflejado en asustarse frecuentemente, en dificultades para concentrarse o conciliar el sueño, y en una marcada irritabilidad; f) estos síntomas han de haberse experimentado al menos durante un mes; g) y, además de que conllevan un importante malestar, implican una disfunción vital en la medida en que interfieren con el funcionamiento personal, social y laboral de la persona.

De cualquier modo, el TEPT es heterogéneo en lo que a la expresión, severidad y cronicidad de sus síntomas se refiere, si bien son tres las categorías de síntomas comunes a todas las personas que desarrollan el trastorno: la re-experimentación, la evitación y la activación. Y entre las situaciones traumáticas que más frecuentemente se asocian al desarrollo del trastorno cabe destacar aquellas que implican agresiones que han sido causadas de modo intencionado por otros, tales como guerras, atentados terroristas o violencia sexual. No obstante, haber sido víctima de una catástrofe natural o sufrir un accidente de tráfico serio también se constituyen en traumas que pueden formar parte del inicio del trastorno (American National Research Council, 2006). De hecho, para que una situación pueda ser catalogada de traumática es necesario que el individuo la valore cognitivamente como tal, y la interpretación de dicha circunstancia puede depender de factores diversos, tales como la propia historia de cada individuo o del marco cultural al que pertenezca (Browman y Yehuda, 2004; McNally, 2004). De cualquier modo, algunos estudiosos del TEPT consideran que el trastorno es una respuesta psicobiológica a un trauma contundente y, por tanto, no sujeta a la cultura o circunstancias sociales del individuo (Yehuda y McFarlane, 1997). Sea como fuere, la exposición a una situación traumática es una condición necesaria pero no suficiente para desarrollar un TEPT; consiguientemente, son las características de las personas las que explican que exista una mayor probabilidad a desarrollar el trastorno (McFarlane, 1999; Yehuda, McFarlane y Shalev, 1998). En este sentido, dichas características son de dos tipos: variables psicológicas y variables de índole biológicas.

Entre las primeras, se han estudiado variables como el neuroticismo (Breslau, Davis y Andreski, 1995; Davidson, Kudler y Smith, 1987; Kuhne, Orr y Barage, 1993); el tipo de creencias características de estos individuos tales como la percepción de vulnerabilidad o fortaleza, la percepción de control sobre el acontecimiento y la creencia en torno a si un acontecimiento es o no peligroso (Ehlers y Clark, 2000; Parrot y Howes, 1991) y las valoraciones de miedo y daño (Alvarez-Conrad, Zoellner y Foa, 2001; Ehlers, Mayou y Bryant, 2003), entre las que cabe destacar la consideración de la ansiedad como dañina, como establece el constructo de sensibilidad a la ansiedad (Asmundson, Bonim y Frombach, 2000; Fedoroff, Taylor, Asmundosn y Koch, 2000; McNally et al., 1987; Orr, McNally, Rosen y Shalev, 2004; Reiss y McNally, 1985; Schmidt, Zvolensky y Maner, 2006).

Se ha venido postulando que, tanto las características psicológicas de las personas aquejadas de TEPT, como los propios síntomas definitorios del trastorno, pueden ser los factores explicativos de los peores niveles de salud física de estos pacientes (Hoge, Terhakopian, Castro, Messer y Engel, 2007; 
Miranda, Meyerson, Marx y Tucker, 2002; Sareen, Cox, Stein, Afífi, Fleet y Asmundson, 2007). De un lado, porque estos factores psicológicos parecen afectar a la percepción de la propia salud de estos individuos, comparados con población sana o aquejada de otros problemas psicológicos, indicando que estas personas se consideran a sí mismas menos sanas y, consiguientemente, hacen un mayor uso de los servicios de salud (Zoellner, Goodwin y Foa, 2000). De otro, porque el propio trastorno y sus características implica comportamientos de riesgo para la salud que incrementan los síntomas asociados a su pérdida (Dobie et al., 2007; Schnurr y Spiro, 1999).

Con relación a los primeros, existe evidencia empírica indicativa de que el nivel de activación (arousal) característico del TEPT predice la percepción de síntomas somáticos indicativos de una peor salud (v.gr. Hoge et al., 2007; Kimerling, Glum y Wolfe, 2000; The Iowa Persina Gulf Study Group, 1997). No obstante, otros investigadores han obtenido resultados indicativos de que es la severidad de la re-experimentación de los síntomas durante el trauma, y no la hiperactivación, lo que explicaría un incremento en la percepción de síntomas somáticos (Zoellner et al., 2000). Asimismo, otros estudios han mostrado que es la sensibilidad a la ansiedad la podría dar cuenta de estos resultados (Jakupcak et al., 2006), en la medida en que predice la evitación emocional y la hipervigilancia de las sensaciones corporales interoceptivas (Zvolensky y Forsyth, 2002). Concretamente, los hallazgos del estudio de Jakupcak et al. (2006) muestran que la sensibilidad a la ansiedad explica un mayor porcentaje de la varianza de los síntomas físicos percibidos que la severidad de la sintomatología del TEPT. Más aún, sus resultados indican que la sensibilidad a la ansiedad media la relación entre la sintomatología del TEPT y el número de quejas somáticas. De otro lado, se han obtenido resultados indicativos de que la sensibilidad a la ansiedad implica, en algunas personas, la evitación de eventos privados internos desagradables, en el intento de evitar las sensaciones corporales relacionadas con ellos y que el abuso de sustancias se utiliza como medio para escapar de tales situaciones (Forsyth, Parker y Finlay, 2003) lo que, en definitiva, alude a comportamientos de riesgo.

En referencia estas conductas de riesgo como predictores de peores niveles de salud, algunos trabajos evidencian un aumento del consumo de sustancias tóxicas, tales como el alcohol, el tabaco u otro tipo de drogas ilegales en personas diagnosticadas de TEPT (Asmundson, Stein y McCreary, 2002; Beckham et al., 1998; Schnurr y Spiro, 1999). Concretamente, la investigación de Asmundson et al. (2002) evidencia que el abuso de alcohol disminuye los niveles de salud percibida en pacientes con TEPT, si bien el porcentaje de varianza explicada por esta variable es menor a la explicada por los propios síntomas del TEPT.

Atendiendo ahora al segundo tipo de variables consideradas como relevantes en el padecimiento del TEPT, los factores de índole biológica, se ha venido prestando especial atención a los cambios hormonales relacionados con la respuesta de estrés que se produce en la experimentación de una situación traumá- tica. En el TEPT es característica la hipoactividad del eje hipotalámico-pituitario-adrenal (HPA) y un aumento del sistema simpático-adreno-medular (SAM). Esto apunta hacia la posibilidad de que se produzca una desinhibición de las respuestas inflamatorias mediada por los componentes innatos del sistema inmunitario (Rohleder y Karl, 2006; Wessa y Rohleder, 2007). El incremento de las concentraciones de mediadores inflamatorios puede ser consecuencia de las alteraciones en la función del sistema endocrino en respuesta al estrés, concretamente los ejes HPA (Heim, Ehlert, Hellhammer, 2000; Raison y Miller, 2003) y SAM (Geracioti, Baker y Ekhator, 2001).

En lo que al eje HPA se refiere, la mayoría de resultados obtenidos en muestras humanas evidencia una disminución de los niveles de cortisol en plasma tanto en individuos con TEPT como en sujetos expuestos a situaciones traumáticas previas al desarrollo del TEPT (Delahanty, Raimonde, Spoonster, 2000; Yehuda et al., 2000). Resultados similares se han obtenido con medidas de cortisol en saliva (Wessa, Rohleder, Kirschbaum y Flor, 2006; Yehuda, Golier y Kaufman, 2005). No obstante, existen hallazgos que apuntan en la dirección contraria: en los sujetos diagnosticados del TEPT se produce un aumento de los niveles de cortisol en saliva (Lindauer, Olff, van Meijel, Carlier y Gersons, 2006; Young y Breslau, 2004). En cualquier caso, una posible explicación de estas diferencias radica en la duración del trauma: la disminución de cortisol se evidencia en sujetos con TEPT de larga duración, en tanto que el aumento de sus niveles es característico en individuos que han sufrido un acontecimiento traumático relativamente reciente (Wessa y Rohleder, 2007).

Algunos autores han sugerido que la disminución de los niveles de cortisol en individuos con TEPT podría promover un incremento del nivel de vulnerabilidad a padecer enfermedades autoinmunes, inflamatorias, síndromes de dolor crónico, alergias y asma (Heim, Ehlert, Hanker y Hellhammer, 1998). De hecho, estudios efectuados con ratas evidencian que es la falta de corticosterona la que explica la mayor probabilidad de desarrollar artritis inducida inmunológicamente (Sternberg, Hill y Chrousos, 1989).

Respecto al eje SAM, los estudios efectuados muestran en conjunto que los pacientes con TEPT se caracterizan por una hiperactivación noradrenérgica que parece asociarse con la hipervigilancia característica del trastorno (Geracioti et al., 2001; Glover y Poland, 2002; Rohleder, Joksimovic, Wolf y Kirschbaum, 2004). Se ha especulado que este incremento en el nivel de actividad del sistema SAM podría también, a partir de la desinhibición del sistema inflamatorio, estar implicado en la patogénesis de la patología física comórbida al TEPT, argumentando que la desinhibición inflamatoria sería una consecuencia de la severidad del TEPT y que estaría mediada por niveles bajos de cortisol (Wessa y Rohleder, 2007).

En definitiva, los estudios que han investigado la función inmune en sujetos diagnosticados de TEPT señalan hacia la presencia de un sistema de respuesta inflamatorio sobreacti- 
vado. Autores como Rohleder y Karl (2006) concluyen, ante la evidencia empírica disponible, que el mayor número de enfermedades y síntomas físicos característicos del padecimiento del TEPT vendría explicado en parte por la inflamación periférica, las alteraciones del sistema endocrino y el incremento de la actividad inflamatoria. De cualquier modo, estos autores también señalan que las conductas de riesgo para la salud características de muchos sujetos con un TEPT (tales como el abuso de tabaco, de alcohol o de otro tipo de drogas) explicarían también la desinhibición de la respuesta inflamatoria, incrementando el riesgo de aparición de síntomas físicos propios del TEPT, entre ellos el dolor (Wessa y Rohleder, 2007).

Sin lugar a dudas, los resultados relativos a los cambios psicofisiológicos que se producen en sujetos con TEPT son muy sugerentes y podrían justificar, al menos en parte, la mayor presencia de enfermedades físicas en estos pacientes. No obstante, es patente la necesidad de obtener mayor cantidad de evidencia empírica en torno a estos hallazgos, tanto a partir de estudios experimentales de laboratorio como con investigaciones longitudinales.

Relevancia de las variables psicológicas en la explicación de la pérdida de la salud: el dolor crónico como paradigma

El dolor es el síntoma más frecuente en todas las enfermedades. Cuando aparece un dolor agudo, la persona siente daño de manera brusca y relativamente grave, retirando su cuerpo de la fuente de dolor en un acto reflejo: el dolor agudo resulta adaptativo al medio. Pero con demasiada frecuencia, y sin explicación orgánica aparente, el dolor inicial se mantiene -con ligeras fluctuaciones o importantes exacerbaciones-, dejando de ser útil para convertirse en un problema personal, laboral y social, amén de un grave dolencia de salud como la representada por el dolor crónico.

Tal y como Merskey y Bogduk (1994) señalan, la Asociación internacional para el estudio del dolor (International Association for the Study of Pain, IASP) acepta la premisa de que tal patología es, incuestionablemente, una sensación en una parte o partes del cuerpo pero, igualmente, se trata siempre de una experiencia perceptiva y subjetiva desagradable y, por tanto, emocional, resultante de un amplio número de factores: biológicos, psicológicos y sociales. Esta definición pone de manifiesto la evolución que se ha producido en el concepto de dolor: el enfoque biomédico tradicional ha quedado atrás, dando paso a una nueva y más compleja perspectiva multidimensional que tiene en cuenta los tres aspectos de la salud: el biológico, el psicológico y el social. El dolor podría estar asociado con algún problema de enervación (biológico), bien deberse a causas cognitivo-emocionales (psicológicas) o a variables únicamente conductuales. De hecho, la IASP acepta la premisa de que, en ausencia de daño tisular, hay que centrarse en factores psicológicos que subyacen a la percepción de dolor en los sujetos (Morris, 1998).
En las últimas décadas, un número creciente de investigaciones muestra en sus resultados que el miedo y la ansiedad influyen en la experiencia de dolor y, más concretamente, en la discapacidad que el dolor puede provocar. El miedo al dolor, es decir, el grado en el que la persona evita las conductas o experiencias que puedan causar dolor, estaría condicionado por el contexto psicosocial en el que ocurren los eventos dolorosos. En este marco teórico, uno de los intentos más recientes para explicar cómo los pacientes con dolor pueden llegar a alcanzar graves niveles de discapacidad funcional es el modelo del miedo-evitación al dolor de Vlaeyen, Kole-Snijders, Boeren y van Eek (1995).

El término "miedo-evitación" aplicado al ámbito del dolor aparece por vez primera en 1983 de la mano de Lethem, Slade, Troup y Bentley, autores que describen un modelo en el que el miedo al dolor y la subsiguiente evitación, perpetúan de hecho la experimentación de dolor, incluso en ausencia de patología orgánica demostrable. En 1995, y sobre la base de este modelo, algunos autores toman en consideración ciertos factores cognitivos y no sólo los conductuales. Se propone una nueva versión en la que tienen cabida numerosos hallazgos posteriores y la cadena de relaciones entre variables es más compleja (Vlaeyen et al, 1995; Vlaeyen, Kole-Snijders, Rotteveel, Ruesink y Heuts, 1995). El modelo describe conexiones causales entre los distintos elementos que lo componen, desempeñando las variables de miedo y evitación un papel clave en el proceso de cronificación del dolor. El modelo postula dos respuestas opuestas ante el miedo en el contexto del dolor crónico: evitación y afrontamiento; el sujeto tratará de evitar el dolor si lo interpreta como grave amenaza para su integridad física, en cuyo caso tenderá a no moverse y a reducir su actividad hasta que ceda la molestia. Por el contrario, seguirá funcionando con normalidad a pesar del dolor si no repara en su significado ni permite que éste interfiera en su vida cotidiana.

En el primer caso, el miedo al dolor (emoción) y el catastrofismo en su interpretación (cognición) hacen que se incremente el dolor percibido, lo que fomenta un tipo de afrontamiento pasivo (conducta) que lo hacen vulnerable al desuso, la discapacidad y la depresión (Vlaeyen y Linton, 2000). En el segundo caso, los autores apuntan que la ausencia de miedo junto con una valoración justa del nivel de dolor y un rápido afrontamiento activo, pudiera prevenir la aparición de dolor crónico y facilitar la recuperación del sujeto. Específicamente, estos autores argumentan que las experiencias dolorosas, que se intensifican durante el movimiento, pueden fomentar cogniciones catastrofistas en algunas personas y cogniciones más adaptativas en otras. Como consecuencia, los pacientes que muestran pensamientos catastrofistas es más probable que lleguen a tener miedo al dolor.

Posteriormente, el miedo al dolor se materializaría en la evitación de actividades y situaciones y, a largo plazo, llevaría al aumento de la incapacidad y al malestar afectivo reflejado, a menudo, en síntomas depresivos (Philips 1987). A su vez, el 
empeoramiento de la forma física y el estado de ánimo negativo se relacionan con la disminución de los niveles de tolerancia al dolor (Romano y Turner, 1985). Además de la evitación de actividades físicamente amenazantes, la incapacidad puede persistir por otros motivos como el aumento de la atención que recibe el paciente de otras personas o por la evitación de conflictos sociales y responsabilidades debidas al dolor. Frente a esto, los pacientes con cogniciones más adaptativas es más probable que confronten la situación en vez de evitarla, dando lugar a conductas saludables y a una recuperación más rápida (Vlaeyen y Crombez, 1999). Es patente, por tanto, el relevante papel que las cogniciones, y concretamente las catastrofistas, desempeñan en la experiencia de dolor, mostrando ser el catastrofismo una de las variables que con mayor frecuencia incrementa la intensidad del dolor percibido (Buer y Linton, 2002). Además, las cogniciones catastrofistas sobre el dolor han mostrado también ser determinantes de la discapacidad funcional y del empeoramiento del dolor en el curso del padecimiento del mismo (Keefe, Brown, Wallston y Caldwell, 1989). Es interesante hacer notar, igualmente, que el catastrofismo y las creencias sobre miedo y evitación no sólo son característicos de personas que padecen dolor, sino que están presentes también en la población general (sin dolor o con dolor leve/moderado), pudiendo predisponerla a padecer problemas de dolor crónico (Buer y Linton, 2002).

El modelo de miedo y evitación ha sido reformulado posteriormente (Asmundson, Norton y Vlaeyen, 2004). La modificación más sustancial radica en que los autores introducen la ansiedad como una variable diferenciada del miedo al dolor, indicando que la misma estaría relacionada específicamente con la evitación de situaciones que el sujeto puede considerar como potencialmente dañinas y con la hipervigilancia, en tanto que el miedo al dolor tendría lugar ante situaciones concretas, lo que provocaría respuestas de escape. Esta reformulación, por consiguiente, mantiene la consideración del catastrofismo como mecanismo cognitivo que incrementa el miedo al dolor, pero introduce la noción de que es el miedo repetido al dolor lo que acabaría por provocar ansiedad ante el mismo, siendo esta ansiedad la responsable de que se eviten actividades y movimientos que pudieran provocar dolor. La evitación continua de estas circunstancias acabaría así incrementando el nivel de discapacidad del individuo.

En el intento por explicar porqué algunos sujetos aquejados de dolor catastrofizan más que otros, se ha aludido a variables disposicionales como el neuroticismo (Asghari y Nicholas, 1999; BenDebba, Torgerson y Long, 1997) o la sensibilidad a la ansiedad (Asmundson et al., 2000; Reiss y McNally, 1985). Esta última variable ha mostrado, ciertamente, estar relacionada con el miedo y las conductas de evitación al dolor (Asmundson y Norton, 1995; Asmundson et al., 1999; Asmundson y Taylor, 1996). De ahí que la reformulación del modelo de miedo-evitación (Asmundson et al., 2004) incluya la sensibilidad a la ansiedad como un elemento destacado en la experiencia de dolor.
Efectivamente, los resultados de recientes investigaciones evidencian la importancia que la sensibilidad a la ansiedad tiene en el dolor, siendo más relevante que el propio miedo al dolor (Álvarez y Esteve, 2009; Asmundson y Hadjistavropoulos, 2007; Stewart y Asmundson, 2006), que el neuroticismo y que la ansiedad rasgo (Esteve y Camacho, 2008). Asimismo, algunos de los hallazgos empíricos de que se dispone muestran que la sensibilidad a la ansiedad está estrechamente relacionada con la hipervigilancia (Esteve y Camacho, 2008), otro de los elementos considerados en el modelo de miedo-ansiedad-evitación, y que la hipervigilancia afecta de modo directo al nivel de dolor padecido (Roelofs, Peters, Patijn, Schouten y Vlaeyen, 2004).

De otro lado, el importante papel que parece desempeñar la sensibilidad a la ansiedad en el dolor crónico se refleja también en la relación que guarda esta variable con la evitación experiencial, a la que se está comenzando a prestar especial atención en los últimos años. Los estudios que han tenido en consideración la evitación experiencial como variable mediadora en la experiencia de dolor apuntan en una misma dirección: son los individuos que obtienen puntuaciones elevadas en esta variable los que presentan una menor tolerancia al dolor (Marx y Sloan, 2002; Orsillo y Batten, 2005; Feldner et al., 2006). Autores como Orsillo y Batten (2005) postulan que la evitación experiencial puede considerarse como un modo de afrontamiento genérico que los individuos han desarrollado a partir de sus experiencias vitales negativas. Cuando estas mismas personas comienzan a padecer síntomas relacionados con el dolor utilizan dicho modo de afrontamiento, lo que puede conllevar a la cronificación de su patología, en tanto que promueve conductas de miedo y evitación que acaban por incrementar el grado de discapacidad de los pacientes. Y no hay que olvidar que la evitación experiencial ha sido señalada como una de las variables de diferenciación individual en el padecimiento del TEPT (Tull, Gratz, Salters y Roemer, 2004), en la medida en que se hipotetiza que este trastorno tiene más probabilidad de ocurrencia en individuos que, tras haberse visto expuestos a acontecimientos traumáticos, han mostrado evitar pensamientos o recuerdos en torno a dichos acontecimientos.

Interacción entre el TEPT y el dolor crónico: nexos entre la psico y la pato(logía)

La relación entre el padecimiento del TEPT y la presencia de síndromes de dolor crónico ha recibido una atención especial por parte de un gran número de investigadores. Actualmente es incuestionable que la prevalencia de síndromes de dolor crónico es mayor en muestras de pacientes con TEPT que en la población sana (Otis, Keane y Kerns, 2003; Sareen et al., 2007; Shipherd et al., 2007). Del mismo modo, la presencia de TEPT es mayor en muestras de pacientes diagnosticados de problemas de dolor crónico que en muestras de control integradas por sujetos sanos (ver Asmundson et al., 2002), siendo la probabi- 
lidad de desarrollar TEPT cuatro veces mayor en los sujetos con dolor musculoesqueletal que en individuos sanos (Cox y McWilliams, 2002). Asimismo, los datos de que se dispone indican que los pacientes con dolor que padecen más síntomas de TEPT muestran mayores lesiones físicas, mayor deterioro funcional, mayor malestar emocional y hacen un mayor uso de estrategias desadaptativas, en comparación a los pacientes con bajos niveles de sintomatología (Duckworth y Lezzi, 2005). Por otro lado, los síntomas del TEPT han mostrado asociarse también a un peor funcionamiento físico diario, a mayor disfunción causada por el dolor, a más síntomas médicos e intervenciones quirúrgicas, así como a más días en cama por enfermedad (Meltzer-Brody et al., 2007). Igualmente, la presencia de TEPT correlaciona con niveles más elevados de dolor, malestar emocional, interferencia con la vida cotidiana y discapacidad funcional (Sherman, Turk y Okifuji, 2000).

Consiguientemente, puede concluirse que el TEPT y el dolor como patología están relacionados. No obstante, aún se desconoce la naturaleza exacta de dicha relación y los mecanismos que dan cuenta de la misma, si bien se han venido formulando diversas propuestas al respecto. Asimismo, y a la luz de la exposición efectuada previamente, es evidente que ambos desórdenes comparten variables que están presentes bien en su inicio, bien en su mantenimiento. Entre los modelos teóricos que tratan de aunar TEPT y dolor crónico, a partir del análisis de las variables comunes a ambos problemas, cabe destacar la teoría del mantenimiento mutuo (Sharp y Harvey, 2001), la teoría de la vulnerabilidad compartida (Asmundson et al., 2002; Asmundson y Hadjistavropolous, 2006), el modelo de la triple vulnerabilidad (Otis et al., 2003) y el modelo de evitación perpetua (Liedl y Knaevelsrud, 2008). No obstante, ha de insistirse, no se dispone aún de un cuerpo de evidencia empírica contrastada que permita afirmar sin lugar a dudas que los postulados de estas, por otra parte, sugerentes propuestas hayan de ser admitidos sin fisuras. Asimismo, se han propuesto modelos que relacionan ambos desórdenes a partir de los componentes psicobiológicos que los mismos comparten, tal y como postulan McLean, Claw, Abelson y Liberzon (2005) o Stam (2007). En las páginas que siguen se efectuará una breve revisión sobre estas propuestas.

Teoría del mantenimiento mutuo (Sharp y Harvey, 2001). La teoría del mantenimiento mutuo sostiene que ciertos componentes del TEPT mantienen y exacerban los síntomas de dolor y que, del mismo modo, ciertos componentes del dolor crónico mantienen o exacerban los síntomas del TEPT. Entre estos factores se encuentran: (1) los sesgos atencionales, que hacen que los pacientes atiendan a los estímulos amenazantes y dolorosos; (2) la sensibilidad a la ansiedad, que hace al individuo más vulnerable a catastrofizar sobre el trauma y sobre el dolor; (3) el dolor como recordatorio del trauma, de modo que éste dispararía la respuesta de activación que, a su vez, conllevaría a que las situaciones que provocan dolor fueran evitadas; (4) la evi- tación, tanto de estímulos que recordasen el trauma como del propio dolor en sí mismo; (5) la fatiga y el letargo (asociados a la depresión comórbida a ambos trastornos); (6) la ansiedad generalizada y (7) las demandas cognitivas causadas por los síntomas de ambos desórdenes, las cuales podrían limitar el uso de estrategias de afrontamiento adaptativas.

Este modelo señala también que las sensaciones dolorosas experimentadas por un enfermo aquejado de un síndrome de dolor crónico podrían funcionar como un recordatorio del trauma en el que tuvo su origen (puesto que algunos acontecimientos traumáticos, por su naturaleza, provocan dolor físico), disparando la respuesta de activación (uno de los síntomas característicos del TEPT). Esta activación fisiológica promovería la evitación de las actividades que potencialmente provocan dolor y, por tanto, el decondicionamiento físico, lo que aumentaría la probabilidad de experimentar sensaciones dolorosas. La persona quedaría así atrapada en un círculo vicioso, en el cual los síntomas del TEP y del dolor crónico interaccionarían para producir malestar e incapacidad funcional.

Teoría de la vulnerabilidad compartida (Asmundson et al., 2002; Asmundson y Hadjistavropolous, 2006). De acuerdo a esta teoría, existirían diferencias individuales, posiblemente determinadas genéticamente, que predisponen a las personas a desarrollar TEPT y que, al mismo tiempo, predisponen también a los individuos a padecer dolor crónico. Entre dichas características se encontrarían, por ejemplo, la afectividad negativa (entendida como característica de personalidad estable), la evitación del daño, la sensibilidad a la ansiedad y la baja tolerancia a la incertidumbre. De entre todos estos factores, los autores de la teoría de la vulnerabilidad consideran la sensibilidad a la ansiedad como uno de los factores más prometedores a la hora de explicar porqué el TEPT y el dolor co-ocurren. Se basan, para ello, en la evidencia existente acerca de que la sensibilidad a la ansiedad es una variable que amplifica la intensidad de la reacción emocional, particularmente del miedo y de la ansiedad, y en que ha mostrado ser un factor de predisposición de los ataques de pánico (Taylor, 1999). Así, los autores postulan que esta variable también podría ser un factor predisponente al TEPT y al sufrimiento y discapacidad asociados al dolor crónico de origen musculoesquelético (Asmundson, 1999).

Como se expuso anteriormente, en el caso del TEPT, la sensibilidad a la ansiedad provocaría que el grado de alarma causado por el estresor se combinara con la alarma provocada por las sensaciones fisiológicas, lo que exacerbaría la reacción emocional, incrementándose así el riesgo de desarrollar el trastorno. En el caso del dolor crónico, la sensibilidad a la ansiedad incrementaría el miedo y la evitación de actividades que pudieran inducir dolor, lo que aumentaría la probabilidad de que el dolor se mantuviera a lo largo del tiempo. Si el estresor traumático y el evento precipitante del dolor son el mismo u ocurren próximos en el tiempo, la sensibilidad a la ansiedad actuaría amplificando la respuesta ante ambos desórdenes e incremen- 
taría la vulnerabilidad para el desarrollo de ambas condiciones. Además, la sensibilidad a la ansiedad podría incrementar la ansiedad en los sujetos con TEPT, elevando su hipervigilancia y disminuyendo los umbrales de percepción a estímulos dolorosos.

El modelo de la triple vulnerabilidad (Otis et al., 2003). Esta propuesta se basa en el modelo de la triple vulnerabilidad del TEPT (Keane y Barlow, 2002) según el cual la exposición ante una situación que se parece o que simboliza algún aspecto de la situación traumática provoca una respuesta de alarma la cual, por sí sola, no conduciría al TEPT pero que unida a la percepción de incontrolabilidad ante propio evento y la respuesta al mismo, puede acabar desencadenándolo. Otis et al. (2003), tratan de aplicar este modelo al desarrollo del dolor.

Así, según estos autores, al igual que los sentimientos de incontrolabilidad del acontecimiento traumático y sus consecuencias podrían desencadenar un TEPT, los sentimientos de incontrolabilidad ante el padecimiento de dolor podrían conducir al desarrollo de un problema de dolor crónico. Los pacientes que perciben que su dolor es incontrolable e impredecible, desarrollarían sentimientos de baja autoeficacia y se caracterizarían por un marcado afecto negativo, lo que originaría que la persona sintiera miedo a las situaciones que podrían generar dolor y las evitara lo que, a su vez, alimentaría los sentimientos de incontrolabilidad y la baja autoeficacia. Cuando personas con cierta vulnerabilidad (es decir, aquellas predispuestas a ser fisiológicamente lábiles, con una marcada falta de control, que focalizan la atención en su ansiedad y responden ante ella con miedo, por tanto con alto grado de sensibilidad a la ansiedad) son expuestas a un evento que es tanto traumático como doloroso, se incrementaría la probabilidad a que desarrollasen ambos trastornos.

El modelo de evitación perpetua (Liedl y Knaevelsrud, 2008). La propuesta de evitación perpetua se basa, por un lado, en el modelo cognitivo de Ehlers y Clark (2000) formulado para dar explicación del TEP y, por otro, en el modelo de miedo-evitación del dolor (Vlaeyen y Linton, 2000), previamente reseñados.

De acuerdo a sus postulados, las personas con TEPT llevan a cabo un procesamiento cognitivo disfuncional, el cual incrementa la activación física y psicológica. Los recuerdos e imágenes del trauma y las intrusiones (vivenciados como si estuvieran ocurriendo aquí y ahora) incrementan la activación corporal mediante el aumento de la tasa cardíaca, la presión arterial, la tensión muscular y la actividad gastrointestinal. Esta hiperactivación conduce, por un lado, a la evitación de ciertos estímulos y, por otro, al incremento directo de la sensación dolorosa. El incremento de la respuesta de dolor puede reforzar creencias negativas acerca del mismo y el miedo a que las actividades causen dolor, lo que tendría como consecuencia que el sujeto también evitara movimientos o actividades que piensa que pueden provocarle dolor. De este modo, la inactividad de la persona a lo largo del tiempo, resultante de la evitación, podría también incrementar la sensación dolorosa.

Estudios como el de Blechert, Michael, Grossman y Lajtman (2007), arrojan resultados indicativos de que la hiperactivación característica del TEPT se relaciona de modo significativo con un aumento de la actividad simpática, disminuyendo el control cardiaco parasimpático. El papel desempeñado por el sistema simpático en el dolor es también bien conocido. En el estudio efectuado por Gockel, Lindholm, Niemistö y Hurri (2008) se obtuvieron datos reveladores de una asociación significativa entre la variabilidad en el ritmo cardiaco y la discapacidad física percibida. No en vano, Wall y Melzack (1999), pioneros en la formulación del papel desempeñado por las variables psicológicas en la explicación del dolor, ya habían argumentado que la asociación entre el estrés emocional y el incremento de la intensidad del dolor podía ser explicada por el aumento de la actividad del sistema nervioso central, del sistema nervioso autónomo y del sistema musculoesquelético.

La evitación es otra de las variables clave en esta propuesta. Algunos estudios muestran (Nemeroff et al., 2006) que un porcentaje muy elevado de sujetos con TEPT que se habían visto expuestos a una situación altamente traumática, como la representada por un atentado terrorista con numerosas muertes, cumplían los criterios de síntomas de evitación (seis meses después del acontecimiento). Y como se describió en el apartado correspondiente, en el ámbito del estudio del dolor crónico la evidencia acerca del importante papel que la evitación, por miedo al dolor, desempeña en la cronificación del trastorno es innegable.

Formulaciones psicobiológicas explicativas de la interacción entre el TEPT y el dolor crónico. Autores como Stam (2007) señalan que lo que no contemplan las teorías descritas previamente es la posibilidad de que, en el conjunto de individuos vulnerables que desarrollan TEPT, el trauma induzca cambios en los substratos biológicos que alteren tanto los mecanismos de activación como los circuitos de traducción del dolor a nivel cerebral.

Existe evidencia acerca de que el mecanismo de estrés puede influir sobre el procesamiento de dolor; de este modo, los distintos cambios neurológicos producidos tras el trauma en los pacientes vulnerables a desarrollar TEPT podrían hacerlos también más vulnerables a desarrollar dolor crónico (McLean et al., 2005). De hecho, se ha comprobado que cierto tipo de receptores, en concreto los $\mu$-opiáceos, están implicados tanto en la respuesta al estrés como en la supresión de dolor. Concretamente, Liberzon et al. (2007) han encontrado una reducción de la densidad de receptores $\mu$-opiáceos en el córtex cingulado anterior de sujetos diagnosticados de TEPT, al compararlos con un grupo de sujetos sanos y con un grupo de traumatizados sin TEPT. Asimismo, se produce una reducción de dichos receptores en la amígdala y un incremento de los mismos en el córtex orbitofrontal de los sujetos traumatizados que no presen- 
tan TEPT, comparados con los sujetos de los otros dos grupos. Estos autores concluyen que la mayor densidad de receptores observada en los grupos expuestos a eventos traumáticos que no desarrollan TEPT representa una regulación adaptativa en respuesta al trauma, mientras que la menor regulación al alza que se observa en el grupo de TEPT puede reflejar una adaptación insuficiente o inadecuada.

Por otro lado, distintas observaciones psicofisiológicas indican que los pacientes con TEPT tienen un sistema nervioso central hipersensitivo, lo que conlleva un incremento de la respuesta de sobresalto, dificultad para habituarse ante información redundante y una respuesta incrementada ante información novedosa (Orr, Metzger y Pitman, 2002). Es probable, por tanto, que estas características tengan como consecuencia que los sujetos con TEPT sean más sensibles a la percepción de estímulos dolorosos (dado que el dolor es un estímulo desagradable, relevante y novedoso).

Por su parte, McLean et al. (2005) han propuesto un modelo biopsicosocial que enfatiza la importancia de las interacciones entre los procesos neurobiológicos centrales y los factores cognitivo-conductuales en el desarrollo de síndromes de dolor postraumático. Parten del modelo de Vaeyen y Linton (2000), anteriormente reseñado, y sus posteriores reformulaciones (Norton y Asmundson, 2003), e incluyen el papel del mecanismo de la respuesta de estrés en el desarrollo de dolor y del procesamiento emocional que tiene lugar después de un trauma. Si bien los autores asocian el dolor con el TEPT desarrollado tras un accidente de tráfico, sus conclusiones bien podrían extrapolarse al padecimiento de dolor y a las consecuencias emocionales que tienen lugar tras el sufrimiento de otros tipos de situaciones traumáticas. Así, según los postulados de este modelo, el sistema central de control de la respuesta de estrés puede influir tanto sobre el desarrollo del TEPT como sobre el desarrollo de síndromes de dolor crónico (concretamente, síndromes de dolor producido tras colisiones entre automóviles, como pueden ser la fibromialgia y el dolor cervical). Como consecuencia del trauma se produciría una desregulación del sistema de respuesta ante el estrés, la cual interaccionaría con ciertos factores cognitivo-comportamentales (tales como la evitación), que modularían a su vez la actividad de los sistemas neurobiológicos relacionados con el procesamiento del dolor y del estrés, dando lugar a la cronificación del dolor y a los desórdenes emocionales postraumáticos. Más específicamente, las consecuencias físicas y emocionales del trauma, las reacciones emocionales al trauma en sí mismo, a las heridas y a los síntomas experimentados, así como la propia respuesta aguda al estrés (moldeada tanto por factores genéticos como por experiencias traumáticas previas) interaccionarían entre sí, lo que provocaría cambios en las vías centrales de procesamiento, incluidas las relacionadas con el dolor, altamente sensibles a la modulación por parte de distintos inputs cognitivos y emocionales. Todo ello resultaría en una amplificación de la señal dolorosa, la cual interaccionaría a su vez con ciertos comporta- mientos que tiene lugar después del trauma y que amplificarían aún más el dolor, entrando la persona en un círculo de síntomas que se mantendrían entre sí en el tiempo.

McLean et al. (2005) señalan múltiples líneas de evidencia empírica que apoyarían tanto la influencia del mecanismo de respuesta al estrés en la patogénesis del dolor crónico, como las secuelas psicológicas que aparecen después de accidentes de tráfico. De hecho, el TEPT, la fibromialgia y el dolor cervical post-colisión (trastornos que suelen ser desencadenados por accidentes de tráfico o por situaciones traumáticas de distinta naturaleza) comparten rasgos epidemiológicos y clínicos. Existe, además, una estrecha relación entre los síntomas de TEPT y los de dolor, experimentados poco después de un accidente, en aquellos pacientes que desarrollan un dolor cervical post-colisión. Por otro lado, al igual que el TEPT, los síndromes de dolor, tales como la fibromialgia, que aparecen después de un accidente de tráfico se caracterizan por una desregulación del mecanismo de estrés. Por último, dicho mecanismo es capaz de influir sobre el procesamiento del dolor a través de múltiples factores, entre los que se encuentran la respuesta emocional ante el evento traumático, los cambios comportamentales que tienen lugar tras el mismo y la presencia de alteraciones a nivel neurobiológico.

Uno de los mecanismos biológicos propuestos para explicar cómo el estrés podría influir sobre el procesamiento del dolor en víctimas de acontecimientos traumáticos es la presencia de alteraciones a nivel pre-frontal. Una disminución en la inhibición pre-frontal de la actividad de la amígdala y el hipotálamo podría generar alguno de los síntomas del TEPT (Browman y Yehuda, 2004). Asimismo, un funcionamiento anormal de la función pre-frontal también se ha asociado con el catastrofismo ante el dolor (Gracely et al., 2004). Asimismo, las regiones corticales tienen capacidad de influir en la sensibilidad extendida al dolor afectando a las vías moderadoras descendentes, por lo que alteraciones en las mismas podrían influir en la modulación de la sensación dolorosa. La amígdala ha mostrado también capacidad de aumentar y disminuir el dolor (Neugebauer, Li, Bird y Han, 2004). No obstante, este mecanismo no es el único mediante el cual el estrés podría intervenir en el desarrollo de dolor crónico después de un acontecimiento traumático. Otros elementos, como los receptores de glucocorticoides de la espina dorsal, las variaciones en cortisol y la segregación de citoquinas también han sido sugeridos (von Känel et al., 2006).

Otra de las fuentes de evidencia que apoya el modelo de McLean et al. (2005) es el hecho de que ciertos factores cognitivo-conductuales hayan demostrado alterar la percepción del dolor a través de los mecanismos descendentes (Gracely et al., 2004). Asimismo, se ha mostrado que otros factores comportamentales, como la disminución de los niveles de movilidad (propios de pacientes aquejados de dolor crónico), alteran la actividad basal del eje hipotalámico-hipofisiario, conduciendo a más dolor, afatiga y a alteraciones del estado de ánimo (Glass et al., 2004). 
Conclusión

En definitiva, tal y como ha tratado de ponerse de manifiesto en las páginas precedentes, TEPT y dolor crónico, como problemas de salud mental y física, co-ocurren. La investigación hasta ahora disponible, así como las formulaciones teóricas que postulan los mecanismos que subyacen a dicha comorbilidad, muestran que ambos desórdenes están conectados a través de factores cognitivos, afectivos, conductuales y fisiológicos que están en la base de ambos problemas de salud. Parece probable, como ya han sugerido otros autores (Roy-Birne, Smith, Goldberg, Afari y Buchwald, 2004), que la relación entre ambos trastornos sea bidireccional. El dolor podría servir como un estímulo traumático para el desarrollo del TEPT, y la hiperactivación, la intolerancia al estrés y la atención selectiva propia del TEPT podrían exacerbar el dolor, lo que explicaría que los pacientes aquejados de ambas condiciones presenten síntomas más graves y un mayor nivel de discapacidad. No obstante, puesto que la evidencia disponible aún está en un estado incipiente, se requiere continuar investigando en esta línea, en el intento de aportar un mayor conocimiento en torno a los factores responsables y mantenedores de esta comorbilidad.

\section{Referencias}

Álvarez, N. y Esteve, R. (2009). Deshabituación en adictos a la droga y dolor crónico. Psicothema, 21, 199-205.

Alvarez-Conrad, J., Zoellner. L.A. y Foa, E. B. (2001). Linguistic predictors of trauma pathology and physical health. Applied Cognitive Psychology, 15, S159-S170.

American National Research Council (2006). Posttraumatic Stress Disorder: diagnosis and assessment. Washington, DC: National Academies Press.

American Psychiatric Association (1980). Diagnostic and statistical manual of mental disorders ( $3^{\text {a }}$ edición). Washington, DC: American Psychiatric Association.

American Psychiatric Association (2000). Diagnostic and statistical manual of mental disorders (4a edición revisada). Washington, DC: American Psychiatric Association.

Asghari, A., y Nicholas, M. K. (2006). Personality and PainRelated Beliefs/Coping Strategies: A Prospective Study. Clinical Journal of Pain, 22, 10-18.

Asmundson, G.J.G. (1999). Anxiety sensitivity and chronic pain: empirical findings, clinical implications, and future directions. En S. Taylor (Ed.). Anxiety sensitivity: theory, research, and treatment of the fear for anxiety (pp. 269285). New Jersey: Lawrence Erlbaum Associates.

Asmundson, G. J. G., Bonim, M.F. y Frombach, I.K. (2000). Evidence of a disposition toward fearfulness and vulnerability to posttraumatic stress in dysfunctional pain patients. Behaviour Research and Therapy, 38, 801-812.

Asmundson, G. J. G., Coons, M. J., Taylor, S. y Katz, J. (2002). PTSD and the experience of pain: research and clinical im- plications of shared vulnerability and mutual maintenance models. Canadian Journal of Psychiatry, 47, 930- 937.

Asmundson, G. J. G. y Hadjistavropolous, H. D. (2006). Addressing shared vulnerability for comorbid PTSD and chronic pain: a cognitive-behavioral perspective. Cognitive Behavioural Practice, 13, 8-16.

Asmundson, G. J. G., Stein, M.B. y McCreary, D. R. (2002). Posttraumatic stress disorder symptoms influence health status of deployed peacekeepers and nondeployed military personnel. Journal of Nervous and Mental Disease, 190, 807-815.

Asmundson, G. J. G. y Norton, G. R. (1995), Anxiety sensitivity in patients with physically unexplained chronic pain: A preliminary report. Behaviour Research and Therapy, 33, 771-777.

Asmundson, G. J. G., Norton, P. J. y Norton, G. R. (1999). Beyond pain: the role of fear avoidance in chronicity. Clinical Psychology Review, 19, 97-119.

Asmundson, G. J. G., Norton, P. J. y Vlaeyen, J. W. S (2004). Fear-Avoidance model of chronic pain: an overview. En G. J. G. Asmundson, J. W. S., Vlaeyen y G. Crombez (Eds.). Understanding and treating fear of pain (pp.3-24). Oxford: Oxford University Press.

Asmundson, G. J. G. y Taylor, S. (1996). Role of anxiety sensitivity in pain-related fear and avoidance. Journal of Behavioral Medicine, 19, 577-586.

Beckham, J.C., Moore, S.D., Feldman, M.E., Hertzberg, J.R., Kirby, A.C. y Fairbank, J. A. (1998). Helath status, somatiziation, and symptom severity of posttraumatic stress disorder in combat veterans with posttraumatic stress disorder. American Journal of Psychiatry, 155, 1565-1569.

BenDebba, M., Torgerson, W.S. y Long, D.M. (1997). Personality traits, pain duration and severity, functional impairment and psychological distress in patient with persistent low back pain. Pain, 72, 115-125.

Blechert, J., Michael, T., Grossman, P., Lajtman, M. y Wilhelm, F.H. (2007). Autonomic and respiratory characteristics of posttraumatic stress disorder and panic disorder. Psychosomatic Medicine, 69, 935-943.

Boscarino, J.A. (1997). Diseases among men 20 years after exposure to severe stress: implications for clinical research and medical care. Psychosomatic Medicine, 59, 605-614.

Breslau, N., Davis, G.C. y Andreski, P. (1995). Risk factors for PTSD-related traumatic events: a prospective analysis. American Journal of Psychiatry, 152, 529-535.

Browman, M.L. y Yehuda, R. (2004). Risk factors and the adversity-stress model. En G.M. Rosen (Ed.). Posttraumatic stress disorder: issues and controversies (pp. 15-43). Hoboken, NJ: John Wiley \& Sons.

Buer, N. y Linton, S.J. (2002). Fear-avoidance beliefs and catastrophizing: occurence and risk factor in back pain and ADL in the general population. Pain, 99, 485-491.

Commitee on Treatment of Post-Traumatic Stress Disorder (2008). Treatment of post-traumatic stress disorder: an 
assessment of the evidence. Washington, D.C.: National Academy Press

Cox, B.J. y McWilliams, L.A. (2002). Mood and anxiety disorders in relation to chronic pain: evidence from the National Co-morbidity Study. Pain Research Management, 5, 11A.

Cwiker, J., Abdelgani, A., Goldsmith, J. R., Quastel, M. y Yevelson, I. I. (1997). Two-year follow up study of stress-related disorders among inmigrants to Israel from the Chernobyl area. Environmental Health Perspectives, 105, 1545-1550.

Davidson, J., Kudler, H. y Smith, R. (1987). Personality in chronic post-traumatic stress disorder: a study of the Eysenck Inventory. Journal of Anxiety Disorders, 1, 259-300.

Delahanty, D. L., Raimonde, A. J. y Spoonster, E. (2000). Initial posttraumatic urinary cortisol levels predict subsequent PTSD symptoms in motor vehicle accident victims. Biological Psychiatry, 48, 940-947.

Dobie, D. J., Kivlahan, D. R., Maynard, C., Bush, K. R., Davis, T. M. y Bradley, K. A. (2004). Posttraumatic stress disorder in female veterans: association with self-reported health problems and functional impairment. Archives of Internal Medicine, 164, 394-400.

Dobie, D. J., Maynard, C., Kivlahan, D. R., Johnson, K. M., Simpson, T., David, A. C. y Bradley, K. (2004). Posttraumatic Stress Disorder Screening Status is Associated with Increased VA Medical and Surgical Utilization in Women. Journal of General Internal Medicine, 21, 58-64.

Duckworth, M. P. y Lezzi, T. (2005). Chronic pain and posttraumatic stress symptoms in litigating motor vehicle accident victims. Clinical Journal of Pain, 21, 251-261.

Ehlers, A. y Clark, D. M. (2000). A cognitive model of posttraumatic stress disorder. Behaviour Research and Therapy, 38, 319-345.

Ehlers, A., Mayou, R.A. y Bryant, B. (2003). Cognitive predictors of posttraumatic stress disorder in children: results of a prospective longitudinal study. Behaviour Research and Therapy, 41, 1-10.

Feldner, M. T., Hekmat, H., Zvolensky, M. J., Vowles, K. E., Secrist,Z. y Leen-Feldner, E. W.(2006). The role of experiential avoidance in acute pain tolerance: a laboratory test. Journal of Behavioral and Experimental Psychiatry, 37, 146-158.

Forsyth, J. P., Parker, J. y Finlay, C. G. (2003). Anxiety sensitivity, controllability, and experiential avoidance and their relation to drug of choice and addiction severity in a residential sample of substance abusing veterans. Addictive Behaviors, 28, 851-870.

Geracioti, T. D., Baker, D. G. y Ekhator, N. N. (2001). CSF norepinephrine concentrations in post-traumatic stress disorder. American Journal of Psychiatry, 158, 1227-1230.

Glass, J. M., Lyden, A. K., Petzke, F., Stein, P., Whalen G., Ambrose, K., Chrousos, G. y Clauw, D.J. (2004). The effect of brief exercise cessation on pain, fatigue, and mood symptom development in healthy, fit individuals. Journal of Psychosomatic Research, 57, 391- 398.
Glover, D. A. y Poland, R. E. (2002). Urinary cortisol and catecholamines in mothers of child cancer survivors with and without PTSD. Psychoneuroendocrinology, 27, 805-819.

Gracely, R H., Geisser, M. E., Giesecke, T., Grant, M. A., Petzke, F., Williams, D. A. y Claw, D. J. (2004). Pain catastrophizing and neural responses to pain among persons with fibromyalgia. Brain, 127, 835-843.

Gockel, M., Lindholm, H., NIemistö, L. y Hurri, H. (2008). Perceived disability but not pain is connected with autonomic nervous function among patients with chronic low back pain. Journal of Rehabilitation Medicine, 40, 355-358.

Jakupcak, M., Osborne, T., Michael, S., Cook, J., Albrizio, P. y McFall, M. (2006). Anxiety sensitivity and depression: mechanisms for understanding somatic complaints in veterans with Posttraumatic Stress Disorder. Journal of Traumatic Stress, 19, 471-479.

Heim, C., Ehlert, U., Hanker, J.P. y Hellhammer, D. H. (1998). Abuse-related posttraumatic stress disorder and alterations of the hypothalamic-pituitary-adrenal axis in women with chronic pelvic pain. Psychosomatic Medicine, 60, 309-318.

Heim, C., Ehlert, U. y Hellhammer, D. H. (2000). The potential role of hypocortisolism in the pathophysiology of stressrelated bodily disorders. Psychoneuroendocrinology, 25, $1-35$.

Hoge, C.W., Terhakopian, R., Castro, C. A., Messer, S.C. y Engel, C. C. (2007). Association of Posttraumatic Stress Disorder with somatic symptoms, health care visits, and absenteeism among Iraq War veterans. American Journal of Psychiatry, 164, 150-153.

Keane, T. M. y Barlow, D. H. (2002). Posttraumatic Stress Disorder. En D. H. Barlow (ED.) Anxiety and its disorder (pp. 418-453). Nueva York: Guildford Press.

Keefe, F. J., Brown, G. K., Wallston, K. A., y Caldwell, D. S. (1989). Coping with rheumatoid arthritis pain: Catastrophizing as a maladaptive strategy. Pain, 37, 51-56.

Kimerling, R., Clum, G. A. y Wolfe, J. (2000). Relationships among trauma exposure, chronic posttraumatic stress disorder symptoms, and self-reported health in women: replication and extension. Journal of Trauma Stress, 13, 115-128.

Kuhne, A., Orr, S. P. y Barage, E. (1993). Psychometric evaluation of post-traumatic stress disorder: The Multidimensional Personality Questionnaire as an adjunct to the MMPI. Journal of Clinical Psychology, 49, 218-225.

Kulka, R.A., Schlenger, W.E., Fairbank, J.A., Hough, R.L., Jordan, B.K., Marmar, C.R. y Weiss, D.S. 1990. Trauma and the Vietnam War generation: report of findings from the National Vietnam Veterans Readjustment study. Nueva York: Brunner / Mazel.

Lethem, J., Slade, P.D., Troup, J.D.G y Bentley, G. (1983). Outline of a fear-avoidance model of exaggerated pain perceptions. Behaviour Research and Therapy, 21, 401-408.

Liberzon, I., Taylor, S. F., Phan, K. L., Brittin, J. C., Fig, L. M., Bueller, J. A., Koeppe, R. A. y Zubieta, J. (2007). Alte 
red central $\mu$-opiod receptor binding after phychological trauma. Biological Psychiatry, 61, 1030- 1103.

Liedl, A. y Knaevelsrud, C. (2008). Chronic pain and PTSD: the perpetual avoidance model and its treatment implications. Torture, 18, 69-79.

Lindauer, R. J., Olff, M., van Meijel, E. P., Carlier, I. V. y Gersons, B. P. (2006). Cortisol, learning, memory, and attention in relation to smaller hippocampal volume in police officers with posttraumatic stress disorder. Biologycal Psychiatry, 59, 171-177.

Marx, B. P. y Sloan, D. M. (2005). Peritraumatic dissociation and experiential avoidance as predictors of posttraumatic stress symptomatology. Behaviour Research and Therapy, 43, 569-583.

McFarlane, A. C. (1999). Risk factors for the acute biological and psychological response to trauma. In R. Yehuda (Ed.). Risk factors for posttraumatic stress disorder (pp. 163190). Washington, D.C.: American Psychiatric Press.

McFarlane, A. C., Atchinson, M., Rafalowicz, E. y Papay, P. (1994). Physical symptoms in posttraumatic stress disorder. Journal of Psychosomatic Research, 42, 607-617.

McLean, S. A., Claw, D. J., Abalson, J. L. y Liberzon, I. (2005). The development of persistent pain and psychological morbidity after motor vehicle collision: integrating the potential role of stress response systems into a biopsychosocial model. Psychosomatic Medicine, 67, 783-790.

McNally, R.J. (2004). Conceptual problems with the DSM-IV criteria for posttraumatic stress disorder. En G.M. Rosen (Ed.). Posttraumatic stress disorder: issues and controversies (pp. 1-14). Hoboken, NJ: John Wiley \& Sons.

McNally, R. J., Luedke, D. L., Besyner, J. K., Peterson, R. A., Bohm, K. y Lips, O. J. (1987). Sensitivity to stress-relevant stimuli in posttraumatic stress disorder. Journal of Anxiety Disorders, 1, 105-116.

Meltzer-Brody, S., Laserman, J., Zolnoun, D., Steege, J., Green, E. y Teich, A. (2007). Trauma and Posttraumatic Stress Disorder in Women With Chronic Pelvic Pain. Obstetrics and Gynecology, 109, 902-905.

Merskey, H. y Bogduk, N. (1994). Classification of chronic pain. Seattle, W.A.: IASP Press.

Miranda, R., Meyerson, L.A., Marx, B.P. y Tucker, P.M. (2002). Civilian-based Posttraumatic Stress Disorder and physical complaints: evaluation of depression as a mediator. Journal of Traumatic Stress, 15, 297-301.

Morris, G.B. (1998). Changing people from Tender-Minded to Tough-Minded: A pathway for dealing with chronic pain. En S. Klarreich (Ed.). Handbook of organizational Health Psychology. Programs to make the workplace healthier (pp.139-155). California. Psychosocial Press.

Nemeroff, C. B., Bremmer, J.D., Foa, E. B, Mayberg, H.S., North, C.S. y Stein, M.B. (2006). Posttraumatic stress disorder: a state-of-the science review. Journal of Psychiatry Research, 40, 1-21.
Neugebauer, V., Li, W., Bird, G. C. y Han, J. S. (2004). The amygdala and persistent pain. Neuroscientist, 10, 221-234.

Norton, P. J. y Asmundson, G. J. G. (2003). Amending the fearavoidance of chornic pain: what is the role of physiological arousal? The Behavior Therapist, 34, 17-30.

Orr, S.P., McNally, R.J., Rosen, G.M. y Shalev, A.S. (2004). Psychophysiologic reactivity: implications or conceptualizing PTSD. En R. Gerald (Ed.). Posttraumatic stress disorder: issues and controversies (pp. 101-138). Hoboken, NJ: John Wiley \& Sons.

Orr, S. P., Meyerhoff, J. L., Edwards, J.V. y Pitman, R. K. (1998). Heart rate and blood pressure resting levels and responses to generic stressors in Vietnam veterans with Posttraumatic Stress Disorder. Journal of Traumatic Stress, 11, 155-164.

Orsillo, S. M. y Batten, S. V. (2005). Acceptance and Commitment Therapy in the treatment of posttraumatic stress disorder. Behavior Modification, 29, 95-129.

Otis, J. D., Keane, T. M. \& Kerns, R. D. (2003). An examination of the relationship between chronic pain and posttraumatic stress disorder. Journal of Rehabilitation Research and Development, 40, 397- 405.

Parrot, C. y Howes, J. L. (1991). The application of cognitive therapy to posttraumatic stress disorder. En T. M. Vallis, J. L. Howes y P. C. Miller (Eds.). The challenge of cognitive therapy: applications to nontraditional populations ( $\mathrm{pp}$. 85-109). Nueva York: Plenum Press.

Philips, H. C. (1987). Avoidance behaviour and its role in sustaining chronic pain. Behaviour Research and Therapy, 25, 273-279.

Raison, C. L. Y Miller, A. H. (2003). When not enough is too much: the role of insufficient glucocorticoid signalling in the pathophysiology of stress-related disorders. Amercian Journal of Psychiatry, 160, 1554-1565.

Reiss, S. y McNally, R. J. (1985). The expectancy model of fear. En S. Reiss y R. R. Bootzin (Eds.), Theoretical Issues in Behavioral Therapy (pp. 107-121). Nueva York: Academic Press.

Roelofs, J., Peters, M. L., Van der Zijden, M., y Vlaeyen, J.W.S. (2004). Does fear of pain moderate the effects of sensory focusing and distraction on cold-pressor pain in pain free individuals? Journal of Pain, 5, 250-256.

Rohleder, N., Joksimovic, L., Wolf, J. M. y Kirschbaum, C. (2004). Hipocortisolism and increased glucocorticoid sensitivity of pro-inflammatory cytokine production in Bosnian war refugees with posttraumatic stress disorder. Biological Psychiatry, 55, 745-751.

Rohleder, N. y Karl, A. (2006). Role of endocrine and inflammatory alterations in comorbid somatic diseases of post-traumatic stress disorder. Minerva Endocrinologica, 31, 273-288.

Romano, J.M. y Turner, J. A. (1985). Chronic pain and depression: does the evidence support a relationship? Psychological Bulletin, 97, 18-34.

Roy-Byrne, P., Smith, W. R., Goldberg, J., ÇAfari, N y 
Buchwald, D. (2004). Post-traumatic stress disorder among patients with chronic pain and chronic fatigue. Psychological Medicine, 34, 363-368.

Sareen, J., Cox, B. J., Stein, M. B., Afifi, T. O., Fleet, C. y Asmundson, G. J. G. (2007). Physical and mental comorbidity, disability, and suicidal behavior associated with posttraumatic stress disorder in a large community sample. Psychosomatic Medicine, 69, 242-248.

Sawchuk, C. N., Roy-Byrne, P., Goldberg, J., Manson, S., Noonan, C. y Beals, J. (2005). The relationship between post-traumatic stress disorder, depression and cardiovascular disease in an American Indian tribe. Psychological Medicine, 35, 1785-1794.

Schnurr, P.P. y Spiro, A. I. (1999). Combat exposure, PTSD symptoms, and health behaviours as predictors of selfreported physical health in older veterans. Journal of Nervous and Mental Disease, 187, 353-359.

Sharp, T. J. y Harvey, A. G. (2001). Chronic pain and posttraumatic stress disorder: mutual maintenance? Clinical Psychology Review, 21, 857-877.

Shemesh, E., Yehuda, R., Milo, O., Dinur, I. Rudnick, A. y Vered, Z. (2004). Posttraumatic stress, nonadherence, and adverse outcome in survivors of a myocardial infarction. Psychosomatic Medicine, 66, 521-526.

Sherman, J. J., Turk, D. C. y Okifuji, A. (2000). Prevalence and impact of posttraumatic stress disorder-like symptoms on patients with fibromyalgia syndrome. Clinical Journal of Pain, 16, 127-34.

Shipherd, J. C., Keyes, M., Jovanovic, T., Ready, D. J., Baltzell, D., Worley, V., Gordon-Brown, V., Hayslett, C. y Duncan, E. (2007). Veterans seeking treatment for posttraumatic stress disorder: what about comorbid chronic pain? Journal of Rehabilitation Research and Development, 44, 153-166.

Stam, R. (2007). PTSD and stress sensitisation: a tale of brain and body. Part 1: human studies. Neuroscience and Biobehavioral Reviews, 31, 530-557.

Sternberg, E.M., Hill, J.M. y Chrousos, G.P. (1989). Inflammatory mediator-induced hypothalamic-pituitary-adrenal axis activation is defective in streptococcal cell wall arthritissusceptible Lewis rats. Proceedings of the National Academic of Science, 86, 4771-4775.

Stewart, S. H. y Asmundson, G. J. G. (2006) Anxiety sensitivity and its impact on pain experiences and conditions: A state of the art. Behaviour Research and Therapy, 35, 185-188.

Taylor, S. (1999). Anxiety sensitivity: theory, research, and treatment of the fear of anxiety. New Jersey: Lawrence Erlbaum Associates.

The Iowa Persian Gulf Study Group (1997). Self-reported illness and health status among Gulf War veterans: a population-based study. JAMA, 277, 238-245.

Tull, M. T., Gratz, K. L., Salters, K. y Roemer, L. (2004). The role of experiential avoidance in posttraumatic stress symptoms and symptoms of depression, anxiety, and somatiza- tion. Journal of Nervous and Mental Disease, 192, 754-761.

Vlaeyen, J. W. S. y Crombez, G. (1999). Fear of movement/(re) injury, avoidance and pain disability in chronic low back pain patients. Manual Theory, 4, 187-195.

Vlaeyen, J. W. S., Kole-Snijders, A.M. J., Boeren, R.G.B. y Van Eek, H. (1995). Fear of movement/(re)injury in chronic low back pain and its relation to behavioural performance. Pain, 62, 363-372.

Vlaeyen, J. W. S., Kole-Snijders, A. M. J., Rotteveel, A., Ruesink, R. y Heuts, P. H. (1995). The role of fear of movement/(re)injury in pain disability. Journal Occupational Rehabilitation, 5, 235-252.

Vlaeyen, J. W. S. y Linton, S. J. (2000). Fear avoidance and its consequences in chronic musculoskeletal pain: a state of the art. Pain, 85, 317-332.

Von Känel, R., Hepp, U., Kraemer, B., Traeber, R., Keel, M., Mica, L y Schryder, V. (2006). Evidence of low-grade systemic pro-inflammatory activity in patients with posttraumatic stress disorder. Journal of Psychiatry Research, 11, 744-752.

Wall, P. D. y Melzack, R. (1999). Textbook of pain (4th edition). Edinburgo: Churchill Livingstone.

Wessa, M. y Rohleder, N. (2007). Endocrine and inflammatory alterations in post-traumatic stress disorder. Expert Review on Endocrinology Metabolism, 2, 91-122.

Wessa, M., Rohleder, N., Kirschbaum, C. y Flor, H. (2006). Altered cortisol awakening response in posttraumatic stress disorder. Psychoneuroendocrinology, 31, 209-215.

Yehuda, R., Bierer, L. M., Schmeider, J., Aferiat, D. H., Breslau, I. y Dolan, S. (2000). Low cortisol and risk for PTSD in adult offspring of Holocaust survivors. American Journal of Psychiatry, 157, 1252-1259.

Yehuda, R. y McFarlane, A. C. (1997). Introduction. En R. Yehuda y A.C. McFarlane (Eds.). Psychobiology of posttraumatic stress disorder (pp. xi-xv). Nueva York: Academy of Sciences.

Yehuda, R., McFarlane, A. C. y Shalev, A. Y. (1998). Predicting the development of posttraumatic stress disorder from the acute response to a traumatic event. Biological Psychiatry, 44, 1305-1313.

Young, E. A. y Breslau, N. (2004). Cortisol and catecholamines in post-traumatic stress disorder: an epidemiologic community study. Archives of General Psychiatry, 61, 394-401.

Zoellner, L. A., Goodwin, M. L. y Foa, E. B. (2000). PTSD severity and health perceptions in female victims of sexual assault. Journal of Traumatic Stress, 13, 635-649.

Zvolensky, M. J. y Forsyth, J.P. (2002). Anxiety sensitivity dimensions in the prediction of body vigilance and emotional avoidance. Cognitive Therapy and Research, 26, 449-460.

Fecha de recepción: 9 de octubre de 2009 Fecha de aceptación: 9 de noviembre de 2009 